\section{NP34 The HEROs Study Year 3: Engaging Families to Promote Healthy Eating and Activity Behaviors in Early Childhood}

Laura Bellows, PhD, MPH, RD,

laura.bellows@colostate.edu, Colorado State

University, Department of Food Science and Human

Nutrition, Fort Collins, CO 80523-1571;

Susan L. Johnson, PhD, University of Colorado

Anschutz Medical Campus; Morgan McCloskey, MSPH,

Colorado State University; Darcy Thompson, MPH, MD,

University of Colorado Anschutz Medical Campus;

Barbara Chamberlin, PhD, New Mexico State

University; Traci Bekelman, PhD, University of Colorado

Anschutz Medical Campus; Lauren Clark, PhD, University of Utah

Objective: The objective of the HEROs Study (HEalthy EnviROnments Study) is to develop a technology-based, interactive family intervention to promote healthy lifestyles and weight outcomes for young children in the family setting.

Description: The project aims to improve family routines and parent-child eating and physical activity (PA) interactions. Research activities will be conducted through four key objectives: formative research; intervention design; intervention testing; and education.

Evaluation: Year 3 focused on completion of the formative research and initialization of intervention design and plans for intervention testing. Parents and caregivers provided input on four key topic areas: food behaviors and environments (mealtime environment survey, $\mathrm{n}=120$; remote food photography method, $\mathrm{n}=31$ parentchild dyads; parent focus group, $\mathrm{n}=7$ groups, 29 participants); physical activity behaviors and environments (parent interview and parent practices survey; $\mathrm{n}=31$ ); mobile device use (results reported in Year 2); and daily life (ecocultural family interview; $n=30$ ). A mixed methods approach is being utilized to synthesize findings from quantitative and qualitative assessments to provide a holistic understanding of participant behaviors, beliefs, attitudes and values related to each topic area. Intervention mapping is being employed as a framework to integrate formative research findings alongside theory, findings from the literature and previous research outcomes to design the HEROs intervention. Year 3 also included the development and testing of prototypes of nutrition and physical activity mobile applications that will serve as key intervention components.

Conclusions and Implications: The HEROs intervention map will be used as a framework for intervention development. Subsequently in Year 4, an implementation study will be conducted utilizing the Plan-Do-Study-Act (PDSA) framework to test intervention components with a subset of families. Intervention module cycles will be studied to understand which of the key intervention components resonates with and drives participants towards behavior change.

Funding: 2015-68001-23240.

\section{NP35 Use of Engaging Online Videos in Conjunction with New Feeding Content to Enhance a Current EFNEP Program}

Sheryl O. Hughes, PhD, shughes@bcm.edu, Baylor College of Medicine, 1100 Bates Street, Houston, TX 77030; Thomas G. Power, PhD, Washington State University; Susan S. Baker, EdD, Colorado State University; Karen V. Barale, MS, Washington State University; Jane Lanigan, PhD; Louise Parker, PhD; M. Catalina Aragon, MS; Karina Silva Garcia, MS

Objective: The overall purpose of the study is to examine the effectiveness of disseminating child feeding content through the Expanded Food and Nutrition Education Program (EFNEP) in Washington State and Colorado Extension. Two implementation methods are being compared: an in-class implementation where videos and in-class activities were added to the Eating Smart * Being Active (ESBA) curriculum and an online implementation where the participants view online videos and complete online activities after the in-person ESBA classes. The design is a randomized controlled trial (RCT) where participants are randomly assigned to one of the two implementation conditions or to an ESBA only control group. Description: Videos from a previous study were modified and additional footage was added to enhance the newly developed in-person and online child feeding content. Inclass activities and online games were developed to support material in the videos. Participants are parents with a child aged 2-8 years participating in the EFNEP in Colorado and Washington State. The online and in-person curriculum was piloted with 30 low-income parents.

Evaluation: Preliminary results based on the pilot data showed significant increases in parental responsiveness during feeding, child involvement in food preparation, use of measured portions, responsiveness to child satiety cues, and offering new foods. The RCT is currently being conducted in two counties in Colorado and three counties in Washington. To date, 137 parents in Washington and 152 parents in Colorado have been enrolled; 350 parents are expected to participate by the end of the study.

Conclusions and Implications: Extension appears to be an effective way to disseminate feeding information to low-income parents through the EFNEP program. Results from the study will be available for presentation.

Funding: 2015-68001-23311.

\section{NP36 Using the Child as Change Agent for Preventing Childhood Obesity in Rural Georgia: Home Environment Results} Marsha Davis, PhD, University of Georgia; CourtneyBrown,PhD,RDN, courtms@uga.edu, University of Georgia, 116 Rhodes Hall, University of Georgia Health Sciences Campus, Athens, GA 30602; Andrea Scarrow, MAL; Frances McCarty, PhD; Richard Christiana, PhD, Appalachian State University; Richard Lewis, PhD, University of Georgia; Rebecca Mullis, PhD, RD 